Bulletin of the Natural History Museum, 2013, 6: 75-88.

Received 25 Aug 2013; Accepted 03 Nov 2013.

DOI: $10.5937 /$ bnhmb1306075J

UDC: $595.78(497.11)$

\title{
COMPENDIUM OF SERBIAN BUTTERFLIES WITH VERNACULAR NAMES
}

\author{
PREDRAG JAKŠIĆ ${ }^{1}$, ANA NAHIRNIĆ ${ }^{2}$, SONJA PETROVIĆ ${ }^{1}$ \\ ${ }^{1}$ University of Niš, Faculty of Science, Departman for Biology and Ecology, \\ Višegradska 33, 18000 Niš, Serbia, e-mail: jaksicpredrag@gmail.com \\ ${ }^{2}$ University of Belgrade, Faculty of Biology, Studentski trg 16, \\ 11000 Beograd, Serbia.
}

As a result of recent attempts to document butterfly fauna and to create a national database of butterfly distribution in Serbia, a checklist of Serbian butterflies, counting representatives of 199 species with their vernacular names, is presented. The vernacular names of butterflies derivate from previously published data and relevant contemporary publications.

Key words: butterflies, Lepidoptera, Serbia, vernacular names

\section{INTRODUCTION}

The first completed list of Serbian names of butterflies was published in 2008, comprising representatives of 193 species (Jakšić \& Đurić 2008). Since that time, the presence of an additional six butterfly species has been 
recorded in Serbia and certain nomenclatural changes have been carried out. Paperwork from the $19^{\text {th }}$ century which designated the Serbian names of some species has been discovered as well. Similar lists of national terms of butterflies were issued recently in Bosnia and Herzegovina (Lelo 2008), Macedonia (Krpač et al. 2008) and Croatia (Šašić \& Mihoci 2011). In addition, Popović \& Đurić (2011) have prepared a list of butterflies in Serbia with revised Serbian terms.

\section{MATERIAL AND METHODS}

The data for a checklist of Serbian butterflies is based on field surveys and relevant literature (Jakšić \& Đurić 2008, Dincã et al. 2010, Jakšić 2011, Popović \& Đurić 2011, Popović \& Milenković 2011). The systematic order and nomenclature were made according to Karsholt \& Razowski (1996), Van Swaay et al. (2010) and Kudrna et al. (2011). The vernacular names are obtained from literature data from both the $19^{\text {th }}$ century and contemporary publications, including Marinković (1851), Pančić (1864), Dusl (1870), Arsenijević (1879), Kozarac (1888), Belić \& Ivšić (1932), Gradojević (1941), Radovanović (1959), Stanković (1966), Jakšić \& Đurić (2008), Popović \& Milenković (2012) and Popović \& Đurić (2011).

\section{RESULTS}

Earliest written data about insects in Serbia were encountered in "The Chilandar Medical Codex" dating from the $15^{\text {th }}$ and $16^{\text {th }}$ centuries. Data significant for humans on arthropods and insects such as scorpions, spiders, lice and bees, and including the silkworm Bombyx mori (Linnaeus, 1758), can be encountered in the text. First published data with Serbian terms of butterflies include those from the mid- $19^{\text {th }}$ century as well as more recent data (Tab. 1).

Respecting priority rules, we have striven to implement those terms in the current list of Serbian butterflies although there has been some compromise where the Serbian term is not precisely determined. This consistency is necessary to avoid and it was already indicated by Belić \& Ivšić (1932), including variety of terminology and zoological nomenclature; thus one term only should be prescribed for one term or subject. On the basis of that procedure the systematic list of Serbian butterflies are shown in Table 2. 
Table 1. - Vernacular names of Serbian butterflies obtained from Serbian literature: 1. Marinković (1851), 2. Pančić (1864), 3. Dusl (1870), 4. Arsenijević (1879), 5. Kozarac (1888), 6. Belić \& Ivšić (1932), 7. Gradojević (1941), 8. Radovanović (1959), 9. Stanković (1966), 10. Jakšić \& Đurić (2008), 11. Popović \& Đurić (2011).

\begin{tabular}{|c|c|}
\hline Scientific name & Vernacular name \\
\hline Parnassius apollo (L., 1758) & $\begin{array}{l}\text { Apolo }(1,2,3,5) \text {; Apolonov leptir }(6,8) \text {, } \\
\text { Apolon }(10,11)\end{array}$ \\
\hline Iphiclides podalirius (L., 1758) & $\begin{array}{l}\text { Vetrilo (1), Jedraš (3), Jedarce (6), } \\
\text { Jedrilac }(10,11)\end{array}$ \\
\hline Papilio machaon (L., 1758) & $\begin{array}{l}\text { Lastin rep }(1,2,3,4,8) \\
\text { Lastin repak }(5,6,9,10,11)\end{array}$ \\
\hline $\begin{array}{l}\text { Anthocharis cardamines } \\
\text { (L., 1758) }\end{array}$ & Zorica $(6,10,11)$ \\
\hline Aporia crataegi (L., 1758) & $\begin{array}{l}\text { Glogovnjak (1), Glogov beli leptir (4), } \\
\text { Glogovac }(6,7,8,9,10,11)\end{array}$ \\
\hline Pieris brassicae (L., 1758) & Kupusar $(5,6,8)$, Veliki kupusar $(10,11)$ \\
\hline Colias crocea (Geoffroy, 1785) & Šafranovac $(9,10,11)$ \\
\hline Colias hyale (L., 1758) & Zlatni leptir (6), Zlatni poštar $(10,11)$ \\
\hline Gonepteryx rhamni (L., 1758) & Žutac (4), Limunovac $(10,11)$ \\
\hline Polyommatus icarus (Rott., 1775) & Gladišev plavac $(6,10,11)$ \\
\hline Argynnis paphia (L., 1758) & $\begin{array}{l}\text { Gospođak (5), Sedefni leptir }(6) \\
\text { Obična sedefica }(10,11)\end{array}$ \\
\hline Issoria lathonia (L., 1758) & Sedefni leptir $(9)$, Mala sedefica $(10,11)$ \\
\hline Vanessa atalanta (L., 1758) & $\begin{array}{l}\text { Admiral }(2,3,5,6,8,9,10,11) \\
\text { Lepotić (4) }\end{array}$ \\
\hline Vanessa cardui (L., 1758) & $\begin{array}{l}\text { Stričkov leptir (6), Čkaljevac }(8,11) \text {, } \\
\text { Lisica (9), Stričkovac (10) }\end{array}$ \\
\hline Aglais io (L., 1758) & $\begin{array}{l}\text { Paunac (1), Dnevni paun }(3) \text {, } \\
\text { Dnevni paunovac }(5,6,8,9,10,11) \text {, } \\
\text { Dnevni paunjac (4) }\end{array}$ \\
\hline Aglais urticae (L., 1758) & Mali koprivar (6), Koprivar $(8,10,11)$ \\
\hline Nymphalis antiopa (L., 1758) & $\begin{array}{l}\text { Mrtvački plašt }(3,6,8), \text { Kadivac }(4), \\
\text { Kraljevski plašt }(10), \text { Kraljev plašt }(11)\end{array}$ \\
\hline N. polychloros (L., 1758) & $\begin{array}{l}\text { Veliki koprivar }(6) \\
\text { Mnogobojac }(7,10,11)\end{array}$ \\
\hline Apatura iris (L., 1758) & $\begin{array}{l}\text { Talasnjak (3), Modri prelivac (10), } \\
\text { Veliki prelivac (11) }\end{array}$ \\
\hline $\begin{array}{l}\text { Coenonympha pamphilus } \\
\text { (L., 1758) }\end{array}$ & Mali satir (9), Mala cenonimfa $(10,11)$ \\
\hline Hipparchia fagi (Scopoli, 1763) & Veliki satir (9), Šumska hiparhija $(10,11)$ \\
\hline
\end{tabular}


Table 2. - Systematic list of Serbian butterflies with vernacular names. Taxonomy and nomenclature follows Karsholt \& Razowski (1996), Van Swaay et al. (2010) and Kudrna et al. (2011).

\begin{tabular}{|c|c|c|}
\hline No. & Scientific name & Serbian name \\
\hline & \multicolumn{2}{|l|}{ LEPIDOPTERA } \\
\hline & \multicolumn{2}{|l|}{ HESPERIOIDEA } \\
\hline & HESPERIIDAE & SKELARI \\
\hline 1 & Erynnis tages (Linnaeus, 1758) & Tamni skelar \\
\hline 2 & Carcharodus alceae (Esper, 1780) & Slezov skelar \\
\hline 3 & Carcharodus lavatherae (Esper, 1783) & Čistac \\
\hline 4 & Carcharodus flocciferus (Zeller, 1847) & Totrljanov skelar \\
\hline 5 & Spialia phlomidis (Herrich-Schäffer, 1845) & Srebrna hesperida \\
\hline 6 & Spialia orbifer (Hübner, 1823) & Dinjicina hesperida \\
\hline 7 & Muschampia proto (Ochsenheimer, 1808) & Pelinova hesperida \\
\hline 8 & Muschampia cribrellum (Eversmann, 1841) & Mozaična hesperida \\
\hline 9 & Pyrgus carthami (Hübner, 1813) & Ribar \\
\hline 10 & Pyrgus sidae (Esper, 1784) & Lipicina hesperida \\
\hline 11 & Pyrgus andromedae (Wallengren, 1853) & Alpijska hesperida \\
\hline 12 & Pyrgus malvae (Linnaeus, 1758) & Slezova hesperida \\
\hline 13 & Pyrgus serratulae (Rambur, 1839) & Zagasiti zujavac \\
\hline 14 & Pyrgus armoricanus (Oberthür, 1910) & Zujavac \\
\hline 15 & Pyrgus alveus (Hübner, 1803) & Proseda hesperida \\
\hline 16 & Pyrgus trebevicensis (Warren, 1926) & Brodar \\
\hline 17 & Heteropterus morpheus (Pallas, 1771) & Karirani skelar \\
\hline 18 & Carterocephalus palaemon (Pallas, 1771) & Helenina hesperida \\
\hline 19 & Thymelicus lineola (Ochsenheimer, 1808) & Smeđi skelar \\
\hline 20 & Thymelicus sylvestris (Poda, 1761) & Srebrni skelar \\
\hline 21 & Thymelicus acteon (Rottemburg, 1775) & Travar \\
\hline 22 & Hesperia comma (Linnaeus, 1758) & Livadski skelar \\
\hline \multirow[t]{3}{*}{23} & Ochlodes sylvanus (Esper, 1777) & Riđi skelar \\
\hline & PAPILIONOIDEA & \\
\hline & PAPILIONIDAE & JEDRILCI \\
\hline 24 & $\begin{array}{l}\text { Zerynthia polyxena (Denis \& Schiffermüller, } \\
1775 \text { ) }\end{array}$ & Uskršnji leptir \\
\hline
\end{tabular}




\begin{tabular}{|c|c|c|}
\hline No. & Scientific name & Serbian name \\
\hline 25 & Zerynthia cerisy (Godart, 1824) & Mirin leptir \\
\hline 26 & Parnassius mnemosyne (Linnaeus, 1758) & Mnemozine \\
\hline 27 & Parnassius apollo (Linnaeus, 1758) & Apolo \\
\hline 28 & Iphiclides podalirius (Linnaeus, 1758) & Vetrilo (Jedrilac) \\
\hline \multirow[t]{2}{*}{29} & Papilio machaon (Linnaeus, 1758) & Lastin rep \\
\hline & PIERIDAE & BELCI \\
\hline 30 & Leptidea sinapis (Linnaeus, 1758) & Gorušičin belac \\
\hline 31 & Leptidea juvernica Williams, 1946 & Zagasiti belac \\
\hline 32 & Leptidea duponcheli (Staudinger, 1871) & Balkanski belac \\
\hline 33 & Leptidea morsei (Fenton, 1881) & Fruškogorski belac \\
\hline 34 & Anthocharis cardamines (Linnaeus, 1758) & Zorica \\
\hline 35 & Anthocharis gruneri (Herrich-Schäffer, 1851) & Mala zorica \\
\hline 36 & Euchloe ausonia (Hübner, 1804) & Čipkasti belac \\
\hline 37 & Aporia crataegi (Linnaeus, 1758) & $\begin{array}{l}\text { Glogovnjak } \\
\text { (Glogovac) }\end{array}$ \\
\hline 38 & Pieris brassicae (Linnaeus, 1758) & Veliki kupusar \\
\hline 39 & Pieris mannii (Mayer, 1851) & Dalmatinski kupusar \\
\hline 40 & Pieris rapae (Linnaeus, 1758) & Mali kupusar \\
\hline 41 & Pieris ergane (Geyer, 1828) & Planinski kupusar \\
\hline 42 & Pieris napi (Linnaeus, 1758) & Žiličasti kupusar \\
\hline 43 & Pieris balcana (Lorković, 1968) & Balkanski kupusar \\
\hline 44 & Pontia edusa (Fabricius, 1777) & Zeleni kupusar \\
\hline 45 & Colias erate (Esper, 1805) & Stepski poštar \\
\hline 46 & Colias crocea (Geoffroy, 1785) & Šafranovac \\
\hline 47 & Colias myrmidone (Esper, 1781) & Zanovetak \\
\hline 48 & Colias caucasica (Staudinger, 1871) & Kavkaski poštar \\
\hline 49 & Colias hyale (Linnaeus, 1758) & Zlatni poštar \\
\hline 50 & Colias alfacariensis (Ribbe, 1905) & Zlatna osmica \\
\hline \multirow[t]{2}{*}{51} & Gonepteryx rhamni (Linnaeus, 1758) & Žutac (Limunovac) \\
\hline & RIODINIDAE & PEGAVCI \\
\hline 52 & Hamearis lucina (Linnaeus, 1758) & Smeđi pegavac \\
\hline
\end{tabular}




\begin{tabular}{|c|c|c|}
\hline No. & Scientific name & Serbian name \\
\hline & LYCAENIDAE & PLAVCI \\
\hline 53 & Lycaena phlaeas (Linnaeus, 1761) & Vatreni dukat \\
\hline 54 & Lycaena helle (Denis \& Schiffermüller, 1775) & Ljubičasti dukat \\
\hline 55 & Lycaena dispar (Haworth, 1802) & Veliki dukat \\
\hline 56 & Lycaena virgaureae (Linnaeus, 1758) & Dukat \\
\hline 57 & Lycaena tityrus (Poda, 1761) & Bakrenac \\
\hline 58 & Lycaena alciphron (Rottemburg, 1775) & Kiseljakov dukat \\
\hline 59 & Lycaena hippothoe (Linnaeus, 1761) & Dolinski dukat \\
\hline 60 & Lycaena candens (Herrich-Schäffer, 1844) & Balkanski dukat \\
\hline 61 & Lycaena thersamon (Esper, 1784) & Pegavi dukat \\
\hline 62 & Thecla betulae (Linnaeus, 1758) & Brezov dukat \\
\hline 63 & Favonius quercus (Linnaeus, 1758) & Hrastov repkar \\
\hline 64 & Callophrys rubi (Linnaeus, 1758) & Kupinov repkar \\
\hline 65 & Satyrium w-album (Knoch, 1782) & Šumski repkar \\
\hline 66 & Satyrium pruni (Linnaeus, 1758) & Trnjinar \\
\hline 67 & Satyrium spini (Denis \& Schiffermüller, 1775) & Trnjinin repkar \\
\hline 68 & Satyrium ilicis (Esper, 1779) & Hrastovčić \\
\hline 69 & Satyrium acaciae (Fabricius, 1787) & Mali repkar \\
\hline 70 & Lampides boeticus (Linnaeus, 1767) & Dugorepi selac \\
\hline 71 & Leptotes pirithous (Linnaeus, 1767) & Kratkorepi selac \\
\hline 72 & Cupido minimus (Fuessly, 1775) & Maleni plavac \\
\hline 73 & Cupido osiris (Meigen, 1829) & Ozirisov plavac \\
\hline 74 & Cupido argiades (Pallas, 1771) & Kratkorepi plavac \\
\hline 75 & Cupido decolorata (Staudinger, 1886) & Bledi plavac \\
\hline 76 & Cupido alcetas (Hoffmannsegg, 1804) & Livadski plavac \\
\hline 77 & Celastrina argiolus (Linnaeus, 1758) & Krkovin plavac \\
\hline 78 & Pseudophilotes vicrama (Moore, 1865) & Dušicin plavac \\
\hline 79 & Pseudophilotes bavius (Eversmann, 1832) & Zagasiti plavac \\
\hline 80 & Scolitantides orion (Pallas, 1771) & Žednjakov plavac \\
\hline 81 & Glaucopsyche alexis (Poda, 1761) & Zelenotrbi plavac \\
\hline 82 & Iolana iolas (Ochsenheimer, 1816) & Pucavac \\
\hline 83 & Phengaris arion (Linnaeus, 1758) & Veliki pegavac \\
\hline
\end{tabular}




\begin{tabular}{|c|c|c|}
\hline No. & Scientific name & Serbian name \\
\hline 84 & Phengaris teleius (Bergsträsser, 1779) & Močvarni pegavac \\
\hline 85 & $\begin{array}{l}\text { Phengaris alcon Dennis \& Schiffermüller, } \\
1775\end{array}$ & Mali pegavac \\
\hline 86 & Plebejus pylaon (Fischer, 1832) & Zagasiti kozinac \\
\hline 87 & Plebejus argus (Linnaeus, 1758) & Stooki plavac \\
\hline 88 & Plebejus idas (Linnaeus, 1761) & Idin plavac \\
\hline 89 & Plebejus argyrognomon (Bergsträsser, 1779) & Blistavi plavac \\
\hline 90 & Plebejus optilete (Knoch, 1781) & Borovničar \\
\hline 91 & Aricia eumedon (Esper, 1780) & Zdravčev plavac \\
\hline 92 & Aricia agestis (Denis \& Schiffermüller, 1775) & Čapljinac \\
\hline 93 & Aricia artaxerxes (Fabricius, 1793) & Tamnosmeđi plavac \\
\hline 94 & Aricia anteros (Freyer, 1838) & Alpijski plavac \\
\hline 95 & Cyaniris semiargus (Rottemburg, 1775) & Vizantijski plavac \\
\hline 96 & Polyommatus escheri (Hübner, 1823) & Kozinac \\
\hline 97 & $\begin{array}{l}\text { Polyommatus dorylas (Denis \& } \\
\text { Schiffermüller, 1775) }\end{array}$ & Tirkizni plavac \\
\hline 98 & Polyommatus amandus (Schneider, 1792) & Graoričar \\
\hline 99 & Polyommatus thersites (Cantener, 1835) & Vučarica \\
\hline 100 & Polyommatus icarus (Rottemburg, 1775) & Gledišev plavac \\
\hline 101 & Polyommatus eros (Ochsenheimer, 1808) & Planinski plavac \\
\hline \multirow[t]{2}{*}{102} & Polyommatus daphnis (Denis & Krzavi plavac \\
\hline & \& Schiffermüller, 1775) & \\
\hline 103 & Polyommatus bellargus (Rottemburg, 1775) & Potkovičar \\
\hline 104 & Polyommatus coridon (Poda, 1761) & Srebrnkasti plavac \\
\hline 105 & Polyommatus admetus (Esper, 1783) & Smeđan \\
\hline 106 & Polyommatus ripartii (Freyer, 1830) & Planinski smeđan \\
\hline \multirow[t]{2}{*}{107} & $\begin{array}{l}\text { Polyommatus damon (Denis \& } \\
\text { Schiffermüller, 1775) }\end{array}$ & Damon \\
\hline & NYMPHALIDAE & ŠARENCI \\
\hline 108 & Libythea celtis (Laicharting, 1782) & Koprivićev leptir \\
\hline 109 & Argynnis paphia (Linnaeus, 1758) & Gospođak \\
\hline 110 & $\begin{array}{l}\text { Argynnis pandora (Denis \& Schiffermüller, } \\
1775 \text { ) }\end{array}$ & Pandorina sedefica \\
\hline
\end{tabular}




\begin{tabular}{|c|c|c|}
\hline No. & Scientific name & Serbian name \\
\hline 111 & Argynnis aglaja (Linnaeus, 1758) & Velika sedefica \\
\hline 112 & $\begin{array}{l}\text { Argynnis adippe (Denis \& Schiffermüller, } \\
1775 \text { ) }\end{array}$ & Ljubičina sedefica \\
\hline 113 & Argynnis niobe (Linnaeus, 1758) & Niobina sedefica \\
\hline 114 & Issoria lathonia (Linnaeus, 1758) & Mala sedefica \\
\hline 115 & Brenthis ino (Rottemburg, 1775) & Inova sedefica \\
\hline 116 & Brenthis daphne (Bergsträsser, 1780) & Karirana sedefica \\
\hline 117 & $\begin{array}{l}\text { Brenthis hecate (Denis \& Schiffermüller, } \\
1775 \text { ) }\end{array}$ & Beloglavičar \\
\hline 118 & Boloria eunomia (Esper, 1799) & $\begin{array}{l}\text { Staroplaninska } \\
\text { sedefica }\end{array}$ \\
\hline 119 & Boloria euphrosyne (Linnaeus, 1758) & Prolećna sedefica \\
\hline 120 & Boloria titania (Esper, 1793) & Titania \\
\hline 121 & Boloria selene (Eversmann, 1837) & Biserna sedefica \\
\hline 122 & Boloria dia (Linnaeus, 1767) & Tkačeva sedefica \\
\hline 123 & Boloria pales (Denis \& Schiffermüller, 1775) & Planinska sedefica \\
\hline 124 & Boloria graeca (Staudinger, 1870) & Grčka sedefica \\
\hline 125 & Vanessa atalanta (Linnaeus, 1758) & Admiral (Lepotić) \\
\hline 126 & Vanessa cardui (Linnaeus, 1758) & Stričkovac \\
\hline 127 & Aglais io (Linnaeus, 1758) & Paunac \\
\hline 128 & Aglais urticae (Linnaeus, 1758) & Koprivar \\
\hline 129 & Polygonia c-album (Linnaeus, 1758) & Riđa sedefica \\
\hline 130 & Polygonia egea (Cramer, 1775) & Vojišničar \\
\hline 131 & Araschnia levana (Linnaeus, 1758) & Šumska riđa \\
\hline 132 & Nymphalis antiopa (Linnaeus, 1758) & Mrtvački plašt \\
\hline 133 & Nymphalis polychloros (Linnaeus, 1758) & $\begin{array}{l}\text { Veliki koprivar } \\
\text { (Mnogobojac) }\end{array}$ \\
\hline 134 & Nymphalis xanthomelas (Esper, 1781) & Žutonogi mnogobojac \\
\hline 135 & $\begin{array}{l}\text { Nymphalis vaualbum (Denis \& } \\
\text { Schiffermüller, 1775) }\end{array}$ & Mrki mnogobojac \\
\hline 136 & Euphydryas maturna (Linnaeus, 1758) & Žuti šarenac \\
\hline 137 & Euphydryas aurinia (Rottemburg, 1775) & Močvarni šarenac \\
\hline 138 & Melitaea cinxia (Linnaeus, 1758) & Obični šarenac \\
\hline
\end{tabular}




\begin{tabular}{lll}
\hline No. & Scientific name & Serbian name \\
\hline 139 & Melitaea phoebe (Denis \& Schiffermüller, & Različkov šarenac \\
& 1775) & \\
140 & Melitaea arduinna (Esper, 1783) & Frejerov šarenac \\
141 & Melitaea trivia (Denis \& Schiffermüller, 1775) & Divizmin šarenac \\
142 & Melitaea didyma (Esper, 1778) & Crveni šarenac \\
143 & Melitaea diamina (Lang, 1789) & Mrki šarenac \\
144 & Melitaea aurelia (Nickerl, 1850) & Zlatni šarenac \\
145 & Melitaea athalia (Rottemburg, 1775) & Bokvičin šarenac \\
146 & Melitaea ornata Christoph, 1893 & Kristofov šarenac \\
147 & Limenitis populi (Linnaeus, 1758) & Veliki topolnjak \\
148 & Limenitis camilla (Linnaeus, 1764) & Mali trepetljikar \\
149 & Limenitis reducta (Staudinger, 1901) & Kozolistovac \\
150 & Neptis sappho (Pallas, 1771) & Grahorovac \\
151 & Neptis rivularis (Scopoli, 1763) & Medunikovac \\
152 & Apatura metis Freyer, 1829 & Panonski prelivac \\
153 & Apatura ilia ([Denis \& Schiffermüller], 1775) & Mali prelivac \\
154 & Apatura iris (Linnaeus, 1758) & Talasnjak \\
155 & Kirinia roxelana (Cramer, 1777) & Šumski rešetkar \\
156 & Kirinia climene (Esper, 1783) & Timočki rešetkar \\
157 & Pararge aegeria (Linnaeus, 1758) & Šumski pegavac \\
158 & Lasiommata megera (Linnaeus, 1767) & Zidni okaš \\
159 & Lasiommata petropolitana (Fabricius, 1787) & Planinski okaš \\
160 & Lasiommata maera (Linnaeus, 1758) & Veliki okaš \\
161 & Lopinga achine (Scopoli, 1763) & Draganin okaš \\
162 & Coenonympha rhodopensis Elwes, 1900 & Rodopska cenonimfa \\
163 & Coenonympha arcania (Linnaeus, 1761) & Biserna cenonimfa \\
164 & Coenonympha glycerion (Borkhausen, 1788) & Kestenjasta cenonimfa \\
165 & Coenonympha orientalis (Rebel, 1910) & Ilirska cenonimfa \\
166 & Coenonympha leander (Esper, 1784) & Livadska cenonimfa \\
167 & Coenonympha pamphilus (Linnaeus, 1758) & Mali satir \\
& & (Mala cenonimfa) \\
168 & Pyronia tithonus (Linnaeus, 1767) & Vratar \\
\hline & Aphantopus hyperantus (Linnaeus, 1758) & Okasti smeđaš \\
\hline
\end{tabular}




\begin{tabular}{|c|c|c|}
\hline No. & Scientific name & Serbian name \\
\hline 170 & Maniola jurtina (Linnaeus, 1758) & Volovsko oko \\
\hline 171 & Hyponephele lycaon (Kühn, 1774) & Livadski smeđaš \\
\hline 172 & Hyponephele lupina (Costa, 1836) & Vukov smeđaš \\
\hline 173 & Erebia ligea (Linnaeus, 1758) & Velika erebija \\
\hline 174 & Erebia euryale (Esper, 1805) & Mala erebija \\
\hline 175 & Erebia manto (Denis \& Schiffermüller, 1775) & Prokletijska erebija \\
\hline 176 & Erebia epiphron (Knoch, 1783) & Obična erebija \\
\hline 177 & Erebia orientalis (Elwes, 1900) & Samotna erebija \\
\hline 178 & Erebia aethiops (Esper, 1777) & Okasta erebija \\
\hline 179 & Erebia medusa(Denis \& Schiffermüller, 1775) & Prolećna erebija \\
\hline 180 & Erebia alberganus (De Prunner, 1798) & Staroplaninska erebija \\
\hline 181 & Erebia gorge (Hübner, 1804) & Zagasita erebija \\
\hline 182 & Erebia rhodopensis (Nicholl, 1900) & Rodopska erebija \\
\hline 183 & Erebia ottomana (Herrich-Schäffer, 1847) & Turska erebija \\
\hline 184 & $\begin{array}{l}\text { Erebia cassioides (Reiner \& Hochenwarth, } \\
\text { 1792) }\end{array}$ & Planinska erebija \\
\hline 185 & Erebia pronoe (Esper, 1780) & Crnoruba erebija \\
\hline 186 & Erebia melas (Herbst, 1796) & Crna erebija \\
\hline 187 & Erebia oeme (Hübner, 1804) & Masliničar \\
\hline 188 & Erebia pandrose (Borkhausen, 1788) & Snežna erebija \\
\hline 189 & Melanargia galathea (Linnaeus, 1758) & Šah-tabla \\
\hline 190 & Melanargia larissa (Geyer, 1828) & Balkanska šah-tabla \\
\hline 191 & Satyrus ferula (Fabricius, 1793) & Veliki satir \\
\hline 192 & Minois dryas (Scopoli, 1763) & Modrooki satir \\
\hline 193 & Hipparchia fagi (Scopoli, 1763) & Šumska hiparhija \\
\hline 194 & Hipparchia syriaca (Staudinger, 1871) & Istočna hiparhija \\
\hline 195 & $\begin{array}{l}\text { Hipparchia volgensis (Mazochin- } \\
\text {-Porshnjakov, 1952) }\end{array}$ & Balkanska hiparhija \\
\hline 196 & Hipparchia statilinus (Hufnagel, 1766) & Jesenja hiparhija \\
\hline 197 & $\begin{array}{l}\text { Arethusana arethusa (Denis \& } \\
\text { Schiffermüller, 1775) }\end{array}$ & Jesenji livadar \\
\hline 198 & Brintesia circe (Fabricius, 1775) & Šumski vratar \\
\hline 199 & Chazara briseis (Linnaeus, 1764) & Samotnjak \\
\hline
\end{tabular}




\section{DISCUSSION}

With respect to the ICZN rules as well as Karsholt \& Razowski (1996), Van Swaay et al. (2010) and Kudrna et al. (2011) the following nomenclatural changes are presented:

Carcharodus floccifera (Zeller, 1847) $>$ Carcharodus flocciferus (Zeller, 1847)

Ochlodes venata (Bremer \& Grey, 1853) > Ochlodes sylvanus (Esper, 1777)

Leptidea reali (Reissinger, 1989) > Leptidea juvernica Williams, 1946

Colias croceus (Fourcroy, 1785) > Colias crocea (Geoffroy, 1785)

Riodininae $>$ Riodinidae

Neozephyrus quercus (Linnaeus, 1758) > Favonius quercus (Linnaeus, 1758)

Maculinea arion (Linnaeus, 1758) > Phengaris arion (Linnaeus, 1758)

Maculinea alcon Dennis und Schiffermüller, $1775>$ Phengaris alcon Dennis und Schiffermüller, 1775

Plebejus pylaon (Fischer von Waldheim, 1832) > Plebejus pylaon (Fischer, 1832)

Vacciniina optilete (Knoch, 1781) > Plebejus optilete (Knoch, 1781)

Polyommatus semiargus (Rottemburg, 1775) > Cyaniris semiargus (Rottemburg, 1775)

Polyommatus eroides (Frivaldszky, 1835) $>$ vrsta nije prisutna u Evropi Proclossiana eunomia (Esper, 1799) > Boloria eunomia (Esper, 1799)

Clossiana euphrosyne (Linnaeus, 1758) > Boloria euphrosyne (Linnaeus, 1758)

Clossiana titania $($ Esper, 1793) $>$ Boloria titania $($ Esper, 1793)

Clossiana selene (Denis und Schiffermüller, 1775) > Boloria selene (Denis \& Schiffermüller, 1775)

Clossiana dia (Linnaeus, 1767) $>$ Boloria dia (Linnaeus, 1767)

Inachis io (Linnaeus, 1758) > Aglais io (Linnaeus, 1758)

Melitaea telona (Fruhstorfer, 1908) > Melitaea ornata Christoph, 1893

Esperarge climene (Esper, 1783) > Kirinia climene (Esper, 1783)

Coenonympha gardetta (De Prunner, 1798) > Coenonympha orientalis (Rebel, 1910)

Hipparchia delattini Kudrna, 1975 > Hipparchia volgensis (Mazochin-Porshnjakov, 1952).

In comparing Serbian names of butterflies with relevant names from neighboring countries of former Yugoslavia, not only certain compatibility but also significant differences have been observed (Tab. 3). 
Table 3. - Comparative review of vernacular names of butterflies from some former Yugoslav countries: Serbia, Bosnia and Herzegovina (Lelo 2008), Macedonia (Krpač et al. 2008) and Croatia (Šašić \& Mihoci 2011).

\begin{tabular}{|c|c|c|c|}
\hline \multicolumn{2}{|c|}{ V E R N A C L A R } & \multicolumn{2}{|l|}{ N A M E S } \\
\hline SERBIA & $\begin{array}{l}\text { BOSNIA AND } \\
\text { HERCEGOVINA }\end{array}$ & MACEDONIA & CROATIA \\
\hline $\begin{array}{l}\text { Carcharodus alceae } \\
\text { SLEZOV SKELAR }\end{array}$ & Sljezov brodar & $\begin{array}{l}\text { Slezova } \\
\text { debeloglavka }\end{array}$ & $\begin{array}{l}\text { Sljezov } \\
\text { debeloglavac }\end{array}$ \\
\hline $\begin{array}{l}\text { Zerynthia polyxena } \\
\text { USKRŠNJI LEPTIR }\end{array}$ & Dijana & $\begin{array}{l}\text { Južno } \\
\text { velikdenče }\end{array}$ & Uskršnji leptir \\
\hline $\begin{array}{l}\text { Parnassius apollo } \\
\text { APOLO }\end{array}$ & Apolon & $\begin{array}{l}\text { Apolonova } \\
\text { peperuda }\end{array}$ & Apolon \\
\hline $\begin{array}{l}\text { Iphiclides podalirius } \\
\text { VETRILO }\end{array}$ & Prugasto jedarce & Edrilec & Prugasto jedarce \\
\hline $\begin{array}{l}\text { Aporia crataegi } \\
\text { GLOGOVNJAK }\end{array}$ & Glogovac & $\begin{array}{l}\text { Glogova } \\
\text { peperuga }\end{array}$ & Glogov bijelac \\
\hline $\begin{array}{l}\text { Pieris brassicae } \\
\text { VELIKI KUPUSAR }\end{array}$ & Veliki bijelac & Golem zelkar & Kupusob bijelac \\
\hline $\begin{array}{l}\text { Colias caucasica } \\
\text { KAVKASKI POŠTAR }\end{array}$ & $\begin{array}{l}\text { Crvenožuti } \\
\text { kupusar }\end{array}$ & $\begin{array}{l}\text { Balkanski } \\
\text { poštar }\end{array}$ & Planinski poštar \\
\hline $\begin{array}{l}\text { Gonepteryx rhamni } \\
\text { ŽUTAC }\end{array}$ & Limunovac & $\begin{array}{l}\text { Žolta (Limon- } \\
\text {-žolta) peperuga }\end{array}$ & Žućak \\
\hline $\begin{array}{l}\text { Lycaena phlaeas } \\
\text { VATRENI DUKAT }\end{array}$ & Mali bakrenac & Mal bakarec & $\begin{array}{l}\text { Mali vatreni } \\
\text { plavac }\end{array}$ \\
\hline $\begin{array}{l}\text { Thecla betulae } \\
\text { BREZOV DUKAT }\end{array}$ & Tekla & $\begin{array}{l}\text { Brezova } \\
\text { peperuga }\end{array}$ & Brezin plavac \\
\hline $\begin{array}{l}\text { Phengaris arion } \\
\text { VELIKI PEGAVAC }\end{array}$ & $\begin{array}{l}\text { Veliki pjegavi } \\
\text { plavac }\end{array}$ & $\begin{array}{l}\text { Krupnodamčest } \\
\text { sinec }\end{array}$ & $\begin{array}{l}\text { Timijanov } \\
\text { plavac }\end{array}$ \\
\hline $\begin{array}{l}\text { Argynnis paphia } \\
\text { GOSPOĐAK }\end{array}$ & Kraljevski mantil & Obična sedefka & Zelena sedefica \\
\hline $\begin{array}{l}\text { Vanessa atalanta } \\
\text { ADMIRAL }\end{array}$ & Admiral & Admiral & $\begin{array}{l}\text { Ljepokrili } \\
\text { admiral }\end{array}$ \\
\hline $\begin{array}{l}\text { Aglais io } \\
\text { PAUNAC }\end{array}$ & Dnevno paunče & $\begin{array}{l}\text { Dneven } \\
\text { paunovec }\end{array}$ & Danje paunče \\
\hline $\begin{array}{l}\text { Aglais urticae } \\
\text { KOPRIVAR }\end{array}$ & Lisičica & Koprivec & Koprivina riđa \\
\hline $\begin{array}{l}\text { Nymphalis antiopa } \\
\text { MRTVAČKI PLAŠT }\end{array}$ & Antiopa & Mrtovečki plašt & Mrtvački plašt \\
\hline $\begin{array}{l}\text { Kirinia roxelana } \\
\text { ŠUMSKI REŠETKAR }\end{array}$ & $\begin{array}{l}\text { Rešetkasti } \\
\text { šarenjak }\end{array}$ & $\begin{array}{l}\text { Šumska } \\
\text { rešetkara }\end{array}$ & $\begin{array}{l}\text { Mediteranski } \\
\text { okaš }\end{array}$ \\
\hline $\begin{array}{l}\text { Maniola jurtina } \\
\text { VOLOVSKO OKO }\end{array}$ & Livadar & Suv list & $\begin{array}{l}\text { Veliko volovsko } \\
\text { oko }\end{array}$ \\
\hline $\begin{array}{l}\text { Melanargia galathea } \\
\text { ŠAH-TABLA }\end{array}$ & $\begin{array}{l}\text { Tamni mramorasti } \\
\text { leptir }\end{array}$ & Šah-tabla & Šahovnica \\
\hline
\end{tabular}




\section{REFERENCES}

Arsenijević, V. (1879): Zoologija za učiteljske i više devojačke škole. - Štamparija A. Pajević, Novi Sad.

Belić, A., Ivšić, S. (1932): Zoološka terminologija i nomenklatura. - Ministarstvo prosvete Kraljevine Jugoslavije. Beograd.

Dincã, V., Kolev, Z., Verovnik, R. (2010): The distribution, ecology and conservation status of the Spinose Skipper Muschampia cribellum (Eversmann, 1841) at the western limit of its range I, Europe Hesperiidae). - Nota Lepidopterologica 33: 39-57.

Dusl, J. (1870): Zoologija za srednje škole. - Državna štamparija. Beograd.

Gradojević, M. (1941): Najvažniji insekti štetni za voćke. - Srpska kraljevska akademija. Beograd.

Jakšić, P. (2011): Butterfly species (Lepidoptera: Hesperioidea and Papilionidea) new to the Serbian fauna. - Biologica Nyssana 2(1): 45-50.

Jakšić, P., Đurić, M. (2008): Srpski nazivi dnevnih leptira (Lepidoptera: Hesperioidea i Papilionoidea). In: Ranđelović, V. (ed.): $9^{\text {th }}$ Symposium of Flora of Southeastern Serbia and Neighbourning Regions, Niš: 231-237. - Univerzitet u Nišu, Prirodno-matematiučki fakultet, Niš.

Karsholt, O., Razowski, J. (1996): The Lepidoptera of Europe A Distributional Checklist. - Apollo Books. Stenstrup.

Kozarac, Đ. (1888): Zoologija za više razrede gimnazije kraljevine Srbije. - Kraljevsko-Srpska državna štamparija, Beograd.

Krpač, V., Krpač, M., Lazarevska, S. (2008): Terminologija od oblasta na zoologijata (Insekti - dnevni peperugi). In: Makedonska terminologija. 113: 1-91. - Makedonska akademija na naukite i umetnostite, Skopje.

Lelo, S. (2008): Dnevni leptiri Bosne i Hercegovine. - Univerzitet u Sarajevu, Prirodno-matematički fakultet, Sarajevo.

Marinković, V. (1851): Jestestvena povjesnica za mladež srbsku. - Beograd.

Pančić, J. (1864): Zoologija po Miln-Edvardsu, Agasicu i Lajnisu. - Državna štamparija, Beograd.

Popovic, M., Milenkovic, M. (2012): First record of Anthocharis gruneri for Serbia (Lepidoptera: Pieridae). - Phegea, Leefdaal (Belgium) 40: 37-38.

Radovanović, M. (1959): Zoologija sa osnovama evolucionizma. - Naučna knjiga, Beograd.

Šašić, M., Mihoci, I. (2011): Annotated checklist of Croatian butterflies with Vernacular names. - Natura Croatica, Zagreb 20(2): 425-436.

Stanković, S. (1966): Biologija - organizacioni tipovi biljaka i životinja za I razred gimnazije. - Zavod za izdavanje udžbenika SR Srbije, Beograd.

Van Swaay, C., Cuttelod, A., Collins, S., Maes, D., López Munguira, M., Šašić., M., Settele, J., Verovnik, R., Verstrael, T., Warren, M., Wiemers, M., Wynhof, I. (2010): European Red List of Butterflies: 1-47. - European Union, Luxembourg. [http://cmsdata.iucn.org/downloads/european_red_list_butterfli-es-_new.pdf] 


\title{
КОМПЕНДИЈУМ ДНЕВНИХ ЛЕПТИРА СРБИЈЕ СА НАРОДНИМ НАЗИВИМА
}

\author{
ПРЕДРАГ ЈАКШИЋ, АНА НАХИРНИЋ, СОЊА ПЕТРОВИЋ
}

\section{Р Е 3 И М Е}

У раду је представљена листа лептира (Lepidoptera: Hesperioidea и Papilionoidea) Србије са њиховим народним именима, која обухвата представнике 199 врста. Сакупљени су и анализирани радови из Панчићевог периода који обухватају народне називе лептира. Поштујући правило приоритета тим старим називима дата је предност док су представници новоконстатованих врста добили своја одговарајућа имена а извршено је и поређење народних назива лептира у Србији, Хрватској, Босни и Херцеговини и Македонији. На основу таксономских и номенклатурних измена, извршено је усаглашавање листе лептира Србије са одговрајућом европском листом. 